\title{
La Escala Latinoamericana y del Caribe sobre Seguridad Alimentaria (ELCSA): Una herramienta confiable para medir
} la carencia por acceso a la alimentación

The Latin American and Caribbean Food Security Scale (ELCSA): a reliable tool to measure lack access to food A Escala América Latina e Caribe Segurança Alimentar (ELCSA): Uma ferramenta confiável para medir a falta de acesso a alimentos

DOI: http://dx.doi.org/10.23913/ricsh.v6i11.118

José Luis Carmona Silva Colegio de Postgraduados Campus Puebla, México jlcarmonas@yahoo.com.mx

Juan Alberto Paredes Sánchez Colegio de Postgraduados Campus Puebla, México Japs52@yahoo.com.mx

\author{
Alfonso Pérez Sánchez \\ El Colegio de Tlaxcala, A.C., México \\ alfonsops65@yahoo.com.mx
}

\section{Resumen}

Descubrir la metodología y herramientas apropiadas para medir la pobreza de manera certera ha sido un objetivo dinámico y perfectible de los investigadores interesados. El cambio conceptual de la medición de la pobreza en México -que ha pasado de ser una consideración unidimensional a una multidimensional basada en derechos sociales universales-, ha dado como resultado que el Consejo Nacional de Evaluación de la Política de Desarrollo Social (CONEVAL) desarrolle toda una metodología de la medición multidimensional de la pobreza, con la que se cuantifica la carencia social de acceso a la alimentación. La medición de esta carencia representa retos específicos ya que su relación con la Inseguridad Alimentaria es manifiesta, y las escalas de seguridad alimentaria basadas 
en experiencias vividas por los integrantes de un hogar, aparecidas a finales de la década de los años ochenta del siglo pasado en Estados Unidos, han demostrado que miden lo que pretenden. La ELCSA es el resultado de múltiples experiencias anteriores con escalas de medición de inseguridad alimentaria en el hogar, validadas tanto en Estados Unidos como Brasil, Colombia, México y otros países de América Latina y el Caribe. México ha adoptado la ELCSA como un instrumento para medir el acceso a la alimentación, uno de los indicadores de carencia social en su metodología para la medición multidimensional de la pobreza.

Palabras clave: Escala Latinoamericana y del Caribe sobre Seguridad Alimentaria, carencia por acceso a la alimentación, medición multidimensional de la pobreza.

\section{Abstract}

Discover the methodology and appropriate tools for measuring poverty in certain way has been a dynamic and perfectible goal of interested researchers. The conceptual change of the measurement of poverty in Mexico - that has gone from being a one-dimensional consideration to a multidimensional universal social rights-based -, has resulted in the National Council for the Evaluation of Social Development Policy (CONEVAL) to develop a whole methodology of the multidimensional poverty measurement, which is quantified social lack of access to food. The measurement of this lack represents specific challenges as their relationship with Food Insecurity is evident, and food security scales based on experiences of the members of a household, appeared at the end of the Decade of the eighties of the last century in the United States, have shown that they measure what they claim to. The ELCSA is the result of multiple previous experiences with scales of measurement of household Food Insecurity, validated in United States such as Brazil, Colombia, Mexico and other countries of Latin America and the Caribbean. Mexico has adopted the ELCSA as an instrument to measure the access to food, one of the indicators of social deficiency in its methodology for the multidimensional poverty measurement.

Key words: Latin American and Caribbean Food Security Scale, lack access to food, multidimensional poverty measurement. 


\section{Resumo}

Descubra a metodologia e ferramentas adequadas para medir a pobreza maneira exata tem sido uma meta dinâmica e perfectível dos pesquisadores envolvidos. A mudança conceitual na medição da pobreza no México, que tem crescido a partir de uma análise unidimensional a um dos direitos sociais universais multidimensionais baseada, resultou no Conselho Nacional de Avaliação da Política de Desenvolvimento Social (CONEVAL) desenvolve toda uma metodologia de medição da pobreza multidimensional, com o qual a falta social do acesso à alimentação é quantificada. Medir essa lacuna representa desafios específicos e sua relação com a insegurança alimentar é manifesto, e escalas de segurança alimentar baseado experimentado por membros de uma experiências domésticas, surgiu no final da década dos anos oitenta do século passado Estados Unidos, mostraram que medida o que eles pretendem. O ELCSA é o resultado de muitas experiências anteriores com escalas de medição insegurança alimentar em casa, validado tanto os EUA eo Brasil, Colômbia, México e outros países da América Latina e do Caribe. México adotou a ELCSA como um instrumento para medir o acesso à alimentação, um dos indicadores de privação social em sua metodologia para medir a pobreza multidimensional.

Palavras-chave: América Latina e Caribe Scale segurança alimentar, a falta de acesso a alimentos, medição multidimensional da pobreza.

Fecha Recepción: Junio $2016 \quad$ Fecha Aceptación: Diciembre 2016

\section{Introducción}

El Consejo Nacional de Evaluación de la Política de Desarrollo Social (CONEVAL) tiene un lema estructuralmente atinado cuyo contenido es irrefutable: "Lo que se mide se puede mejorar".

La dimensión objetiva de cualquier fenómeno aumenta las posibilidades de su comprensión $\mathrm{y}$, por lo tanto, de la toma de decisiones apropiadas, preventivas o correctivas que garanticen su extinción, su decremento o su aumento, según sea el caso.

La Seguridad Alimentaria es una aspiración universal, formalizada cuando la ONU reconoció en 1948 que era un derecho formal de la humanidad, asentado en la Declaración 
Universal de los Derechos Humanos. Los movimientos y acciones que suscitaron esta declaración sólo encontraron eco de forma esporádica en situaciones extremas que se vivieron en algunas regiones de nuestro planeta. La presión, sobre todo de organizaciones no gubernamentales (ONG), llevaron a la Organización de las Naciones Unidas para la Agricultura y la Alimentación (FAO) a convocar a la Cumbre Mundial de la Alimentación celebrada en el año de 1996, de donde surgió la definición más importante sobre Seguridad Alimentaria: "Todas las personas tienen en todo momento acceso físico y económico a suficientes alimentos inocuos y nutritivos para satisfacer sus necesidades alimenticias y sus preferencias en cuanto a los alimentos, a fin de llevar una vida activa y sana" (FAO, 1996). El interés de este trabajo radica en la propuesta de una herramienta confiable que mida la contraparte de la Seguridad Alimentaria, para lo cual la Inseguridad Alimentaria se define como la disponibilidad limitada o incierta de adquirir alimentos adecuados en formas socialmente aceptables. Se sabe que, incluso en los países desarrollados, existen grupos vulnerables que padecen Inseguridad Alimentaria, y que también hay países enteros o grupos mayoritarios en los que persiste con alta prevalencia. Sin embargo, no se tiene certidumbre del número exacto de hogares que padecen este fenómeno, como tampoco el grado de severidad de Inseguridad Alimentaria que enfrentan. Esta circunstancia, sobre todo la toma de conciencia sobre la importancia de combatir adecuadamente este fenómeno, condujo a la búsqueda de un método que de manera científica lo midiera, con el objetivo de identificar más precisamente los grupos de riesgo y de mayor vulnerabilidad para enfrentar el problema con mayor eficacia.

Cuando se habla de Inseguridad Alimentaria el problema no se dimensiona con objetividad ya que parece más conceptual que real; sin embargo, si lo relacionamos con pobreza y sobre todo con pobreza extrema, entonces se delimita mejor: hablamos de personas que no tienen qué comer, de hombres, mujeres y niños que tienen hambre y que no disponen de suficientes alimentos en su hogar. El hambre es la manifestación más abierta de la Inseguridad Alimentaria, la experiencia más excluyente de la sociedad. En esta situación se encuentran más de 800 millones de seres humanos (Simoes, 2001, p. 933); en México, cerca del $25 \%$ de la población no tiene suficiente acceso a la alimentación (CONEVAL, 2012, p. 49). Estas cifras alarmantes impulsan la búsqueda de métodos y herramientas 
confiables y eficaces que midan objetivamente la Inseguridad Alimentaria en grupos o sectores específicos, con el fin de diseñar programas selectivos que la combatan.

\section{Planteamiento}

Hay tres temas importantes a considerar: la carencia por acceso a la alimentación, la medición de dicha carencia, y la Escala Latinoamericana y del Caribe de Seguridad Alimentaria.

El primer tema está íntimamente relacionado con la Seguridad Alimentaria y esta, a su vez, con la pobreza, a tal grado que puede establecerse una relación secuencial: a mayor pobreza corresponde menor Seguridad Alimentaria y si extremamos esta relación diríamos que la pobreza extrema origina el hambre. Este concepto suele ser relegado debido a su crudeza, pero aun así representa el signo más visible, más empírico de la Inseguridad Alimentaria en su máxima expresión.

La razón de ser del concepto de Carencia por Acceso a la Alimentación se remonta a 2001 cuando la Secretaría de Desarrollo Social (SEDESOL) reunió a un grupo de expertos que conformaron el Comité Técnico para la Medición de la Pobreza (CTMP), el cual basándose en la Encuesta Nacional de Ingreso y Gasto de los Hogares del 2000 (ENIGH, 2000) determinó la existencia de tres niveles de pobreza: pobreza de patrimonio, pobreza de capacidades y pobreza alimentaria. Esta metodología se basó en el ingreso de los hogares, de ahí que se considere un modelo unidimensional de la medición de la pobreza.

El 20 de enero de 2004 se promulgó la Ley General de Desarrollo Social (LGDS), cuyo artículo 6 menciona los derechos para el desarrollo social, siendo uno de ellos la alimentación.

La política social intuye la necesidad de idear mecanismos institucionales de evaluación y seguimiento de las políticas de desarrollo social, por lo que se creó el Consejo Nacional de Evaluación de la Política de Desarrollo Social (CONEVAL), organismo que asume como una de sus tareas el cumplimiento del artículo 36 de la LGDS, el cual determina que este consejo debe emitir los lineamientos y criterios para la definición, identificación y medición de la pobreza, considerando un número determinado de indicadores además del ingreso. 
Así, la metodología para la medición multidimensional de la pobreza en México fue elaborada por el CONEVAL con base en la LGDS, en las propuestas de especialistas y en el conocimiento científico y técnico sobre este tema (CONEVAL, 2014). Uno de los indicadores determinantes del carácter multidimensional de la pobreza es el acceso a la alimentación, sin embargo, este concepto es el que más dificultades conceptuales, técnicas y metodológicas presenta para su medición, de ahí que diversos organismos internacionales e instituciones desarrollaran el concepto de Seguridad Alimentaria. Como se ha expuesto, de acuerdo con la FAO (2006), la seguridad alimentaria comprende el acceso en todo momento a comida suficiente para llevar una vida activa y sana. Este concepto de Seguridad Alimentaria se encuentra extremadamente vinculado al de acceso a la alimentación, por lo que se considera que si el primero se mide de manera atinada también se estará midiendo apropiadamente el indicador de carencia a la alimentación.

\section{Medición de la Seguridad Alimentaria}

Partimos de la premisa de que midiendo la Seguridad Alimentaria estamos midiendo la carencia por acceso a la alimentación; revisando los esfuerzos públicos y privados que se han llevado a cabo para diseñar métodos y herramientas que midan de manera veraz la Seguridad Alimentaria, se descubre que son cinco los métodos más usados para evaluar la magnitud de la Inseguridad Alimentaria.

Metodología de la FAO. Fiel a la definición que maneja este organismo, se utiliza un método que mide por separado las cuatro dimensiones de la seguridad alimentaria: disponibilidad, acceso, estabilidad y utilización, cada una con sus propios indicadores. La metodología de la FAO ha sido objeto de amplios debates, pues se basa en la estimación de la ingestión insuficiente de energía alimentaria durante más de un año, pero no puede registrar las fluctuaciones de esta ingesta energética dentro de un mismo año. Por otra parte, es laboriosa, ya que la suma de los indicadores de la seguridad alimentaria pertenecientes a las 4 dimensiones es 31, a cuya totalidad se deben hacer estimaciones (FAO, FIDA, PMA, 2015). 
Este método también requiere de especialistas que lleven a cabo la distribución de frecuencias del consumo de alimentos (expresado como energía alimentaria), que determina el punto límite de la insuficiencia de la ingesta (FAO, 2002).

En resumen, este método se basa en la comparación del consumo habitual de alimentos, expresado en términos de energía alimentaria (Kilocalorías=Kcal), con las necesidades energéticas mínimas (FAO, 2008).

Utilización de encuestas sobre gastos de los hogares para evaluar la inseguridad alimentaria. Esta metodología tiene una cualidad importante, ya que permite obtener informaciones variadas sobre abasto, acceso y diversidad de alimentos, sin embargo, este método aún se puede considerar unidimensional. Por otra parte, el cuestionario que se aplica es demasiado extenso y pormenorizado, además de que tiene el inconveniente de que los costos de recopilación y cómputo en tiempo, recursos financieros y conocimientos técnicos son muy elevados (FAO, 2002). Por último, se piensa que las estimaciones obtenidas pueden llegar a estar sesgadas debido a diversos errores sistemáticos relacionados con el muestreo. Uno de los principales problemas que se atribuyen al trabajo con esas encuestas es que los resultados no son comparables entre países (FAO, 2002, p. 19).

Métodos de encuesta sobre la ingesta individual de alimentos. La principal limitante que se atribuye a este método es la notificación de valores inferiores a los reales, es decir, cuando se trata de este tipo de encuestas se debe lograr un alto grado de colaboración con el sujeto encuestado, además de que se tiende a la "victimización". Otras limitaciones del método son la logística y su costo. Por tanto, se concluye que dicho método es muy útil para la validación de otros métodos más convenientes que evalúan la seguridad alimentaria.

Medición del estado nutricional a partir de datos procedentes de encuestas antropométricas. La FAO debe proporcionar cifras reales sobre la situación y el alcance del problema nutricional para ayudar a los países miembros a que vigilen y den seguimiento a los programas que implementan para combatir el problema de inseguridad alimentaria. En el pasado, uno de los métodos más confiables para medir el estado nutricional eran las mediciones antropométricas, que examinan puntos límites basados en la relación entre los índices antropométricos nutricionales y las deficiencias funcionales, la morbilidad y otros datos que tienen que ver con las consecuencias de la insuficiencia de alimentos. En la 
actualidad, este método ha sido sumamente cuestionado porque se ha descubierto que se encuentra plagado de dificultades relacionadas con la obtención de datos, los cuales no necesariamente reflejan la suficiencia del consumo de alimentos o de la energía porque en ellos influyen otros factores ambientales que intervienen en las medidas antropométricas.

En México, las medidas antropométricas son usadas para medir los estados nutricionales a través de la Encuesta Nacional de Salud y Nutrición (ENSANUT). Sus resultados, además de ser presentados oficialmente, se aprovechan para el análisis de las variables que se manejan (Shamah, Mundo, Rivera, 2014): "La magnitud de la inseguridad alimentaria en México: su relación con el estado de nutrición y factores socioeconómicos".

La ENSANUT obtiene el estado nutricional a base de mediciones de peso y talla o longitud en menores de 5 años. Salta a la vista que este método requiere personal especializado para la obtención de datos confiables, ya que de lo contrario su veracidad estaría en duda. Otro elemento importante es que no existen datos antropométricos sobre adultos.

Mediciones cualitativas de la Inseguridad Alimentaria y el Hambre. En la actualidad, la medición de la (In)Seguridad Alimentaria es una necesidad que se impone como una condición para el combate eficaz contra la pobreza; este tipo de mediciones está basado en Escalas, o como se acostumbra decir en Estados Unidos, Módulos.

Estas mediciones tienen un buen fundamento científico y, una vez concluido el trabajo de la metodología y aplicación de la medición, pueden realizarse y analizarse rápidamente. Tal vez una de las cualidades más importantes de estas mediciones es que la información obtenida proporciona también un concepto de Seguridad Alimentaria fácil de comprender por los responsables de formular políticas. La importancia de las mediciones cualitativas es que incorporan como elementos esenciales la forma en que las personas más afectadas perciben la Inseguridad Alimentaria y el hambre, y por lo tanto, estos métodos cualitativos son mediciones más directas de la Inseguridad Alimentaria.

Si se reflexiona sobre este método de medición se puede observar que existe una óptica diferente de establecer o definir a la población que se encuentra en estado de Inseguridad Alimentaria, porque aunque la fuente de información es la misma, de alguna manera es el investigador el que de acuerdo a sus interpretaciones define quién o quiénes están en inseguridad alimentaria, sin embargo, en este tipo de mediciones son la persona o la familia, sin ningún filtro interpretativo del analista, quienes afirman: "Yo estoy en 
inseguridad alimentaria, yo tengo hambre". Con estas mediciones se obtienen datos importantes sobre la manera como las familias experimentan el hambre y la inseguridad alimentaria.

Una de las discusiones que se presentaron en el Simposio Científico Internacional sobre la medición de la Inseguridad Alimentaria fue que las mediciones cualitativas no deberían denominarse como tales ya que también recopilan información cuantitativa y sus resultados se cuantifican en una forma estadísticamente rigurosa. Por otro lado, se propusieron nombres como: "mediciones directas", "mediciones basadas en la experiencia" o "mediante la experiencia" de la Inseguridad Alimentaria y el hambre (FAO, 2002). Este simposio internacional reconoció que este tipo de mediciones, mediante módulos o escalas, se basa en una metodología que posee aspectos muy positivos en cuanto a validez, pertinencia y utilidad.

La Escala Latinoamericana y del Caribe sobre Seguridad Alimentaria (ELCSA) pertenece a este tipo de mediciones. Este trabajo pretende aportar conocimientos sobre el origen, desarrollo y aplicación en México que ha tenido la ELCSA.

\section{Escala Latinoamericana y del Caribe sobre Seguridad Alimentaria}

La importancia de medir la seguridad alimentaria en estos países con un instrumento cuya metodología de aplicación sea sencilla y barata, radica en que casi todos los países de estas regiones están en vías de desarrollo o aún pueden considerarse subdesarrollados, características que los convierte en lugares donde aún se asientan familias que pueden considerarse campesinas y que, de alguna manera, aunque sea de manera parcial, viven del campo, pero la realidad es que $84 \%$ de las explotaciones familiares abarcan menos de dos hectáreas y ocupan únicamente $12 \%$ de la superficie agrícola, lo cual significa que la mayoría de los agricultores familiares son pobres y que están siendo afectados por la inseguridad alimentaria (FAO, 2014).

Los países latinoamericanos y caribeños que están en esta situación requieren de métodos para medir la inseguridad alimentaria y el hambre a partir de tres cualidades importantes: a) no requieren grandes cantidades de presupuesto, b) su aplicación y obtención de resultados es ágil, lo que puede ser fundamental para los encargados de formular las políticas 
apropiadas, c) genera resultados eficaces a nivel de programas y proyectos para orientarlos hacia objetivos específicos e incluso medir su impacto a plazos determinados.

El fin último que se persigue es la lucha eficaz contra la pobreza y, sobre todo, contra su efecto más dramático: el hambre. En la búsqueda de variables asociadas a la Inseguridad Alimentaria siempre se descubre que el principal determinante de la inseguridad alimentaria en el hogar es la pobreza (Latham, 2002).

\section{¿Qué es la ELCSA?}

La ELCSA es una herramienta de medición directa basada en la experiencia que tienen los hogares sobre la Inseguridad Alimentaria (FAO, 2012). Esta herramienta ha demostrado a lo largo del tiempo que mide lo que quiere medir, lo que le ha valido la aceptación y el reconocimiento de los organismos internacionales, cuya preocupación es precisamente medir la inseguridad alimentaria. La FAO le ha otorgado su reconocimiento y apoyo porque mediante esta herramienta se ha logrado una medición directa que ayuda a comprender el fenómeno de la inseguridad alimentaria, no como una inferencia o deducción sino a través de una confrontación de las experiencias vivenciales que los miembros del hogar han tenido en la expresión más extrema de la pobreza: el hambre. La eficacia y la veracidad de la ELCSA está fuera de dudas, proporciona una dimensión del hambre que se vive en los hogares y, en consecuencia, una medida real de la inseguridad alimentaria.

La ELCSA no es una herramienta de moda, su valor científico ha sido suficientemente comprobado a través de las múltiples validaciones a las que ha sido sometida por parte de investigadores de diversos países latinoamericanos. Para los diferentes gobiernos de la región, que incluso están adoptando la ELCSA para aplicarla a nivel nacional, también ha sido importante ya que sus resultados les permiten tomar decisiones de tipo preventivo o correctivo en relación con la Inseguridad Alimentaria. Esta se puede diagnosticar mediante la propia ELCSA y, por lo tanto, implementar programas de desarrollo apropiados de manera selectiva para, posteriormente, evaluar el impacto de los programas.

Otra cualidad importante de la ELCSA es que no mide de manera general o confusa el problema de la inseguridad alimentaria, sino que permite dimensionarla en tres niveles de inseguridad o en un estado de seguridad alimentaria: Seguridad Alimentaria, Inseguridad Alimentaria Leve, Inseguridad Alimentaria Moderada, e Inseguridad Alimentaria Severa. 
Los países involucrados pertenecientes a la región Latinoamericana y del Caribe están adoptando la ELCSA ya que les permite de manera confiable medir periódicamente su situación real en lo que se refiere a la inseguridad alimentaria, no sólo con un número, sino también en toda su crudeza. Descubre situaciones, preocupaciones y percepciones sobre la falta de alimentos no como un concepto o un elemento estadístico más, sino como una vivencia cotidiana dolorosa y desesperante.

\section{Antecedentes de la ELCSA}

El intento específico de medir la inseguridad alimentaria mediante la existencia de hambre en los hogares, se remonta a 1984 cuando una dependencia gubernamental de Estados Unidos reportó la existencia de grupos de personas que padecían hambre, sin embargo, no se contaba con información suficiente sobre las características de estos grupos, ni con una cuantificación que, de alguna manera, dimensionara el problema (FAO, Ohio State University, 2012).

Concretamente, las escalas de medición de la seguridad alimentaria, tal y como se conocen en la actualidad, tuvieron su origen en un reporte para el monitoreo nacional en nutrición e investigación relacionada (National Nutrition Monitoring and Related Research Act). Este reporte tiene como principal objetivo buscar la estandarización de instrumentos de medición de la inseguridad alimentaria, de tal manera que el país cuente con medidas confiables y no dispersas que permitan la medición y el monitoreo del problema (Hamilton et al., 1997, pp. 1- 13). Del reporte surgen dos instrumentos que son las fuentes de las escalas de medición de la seguridad alimentaria: el Proyecto Comunitario para Identificar Hambre en la Niñez: Community Childhood Hunger Identification Proyect (CCHIP) y la Escala de Radimer/Cornell (Radimer, Olson, Greene, Campbell, Habicht, 1992). Estos dos instrumentos son aprovechados para estructurar la primera escala formalmente sistematizada y así medir la inseguridad alimentaria en Estados Unidos: Household Food Security Supplemental Module (HFSSM). Con este instrumento se elabora desde 1995 un reporte anual sobre la situación de inseguridad alimentaria en ese país (Hamilton et al., 1997). 
La influencia que tuvo la HFSSM como herramienta apropiada para medir la inseguridad alimentaria fue grande, así que, junto con otras escalas fundamentadas en ella, fue adaptada a otros países ya que se trataba de un instrumento sencillo y económico.

Los países que primero adaptaron sus escalas, aprovechando la experiencia de Estados Unidos, fueron los sudamericanos, por ejemplo, Brasil, que generó su propia escala (EBIA) tomando como base la HFSSM. Otros países, como Ecuador, no crearon su propia escala, sino que adoptaron la HFSSM y la sometieron a procesos o estudios de validación (Hackett, Melgar, Álvarez, 2007); México (Melgar et al., 2005); Bolivia (Melgar, 2006); Argentina (Fiszbein, 2003). Incluso en Canadá se utiliza una versión equivalente a la HFSSM para evaluar y monitorear la inseguridad alimentaria (Health Canadá, 2004).

Por otra parte, el índice CCHIP (proyecto-propuesta), nacido en Massachusets también ha sido tomado como base para generar otras escalas en países sudamericanos como Venezuela, donde se creó una escala propia inspirada en el CCHIP para realizar estudios de inseguridad alimentaria en hogares periurbanos (Lorenzana, Mercado, 2002). Aprovechando la experiencia venezolana, Colombia también creó su escala (Álvarez, Estrada, Melgar, 2006) para medir la inseguridad alimentaria doméstica.

Los estudiosos en el tema se han esforzado por evitar la diversificación y encontrar homogeneidad a partir de las experiencias derivadas del índice CCHIP en Venezuela y Colombia, así como las de Radimer/Cornell, HFSSM y la Escala Brasileña (EBIA). La necesidad de un instrumento unificador generó un proceso evolutivo natural de intercomunicación con investigadores latinoamericanos, cuyos esfuerzos e intereses comunes por la búsqueda del intercambio de experiencias con el propósito de promover el desarrollo de una escala única para la medición de la (In)seguridad alimentaria en los hogares de América Latina y el Caribe, dio como resultado la Escala Latinoamericana y del Caribe sobre Seguridad Alimentaria (ELCSA), cuyas bases inmediatas son la Escala Lorenzana Colombiana, la Escala Brasileña EBIA y los elementos de la propia HFSSM. 


\section{Figura 1. Evolución ELCSA}

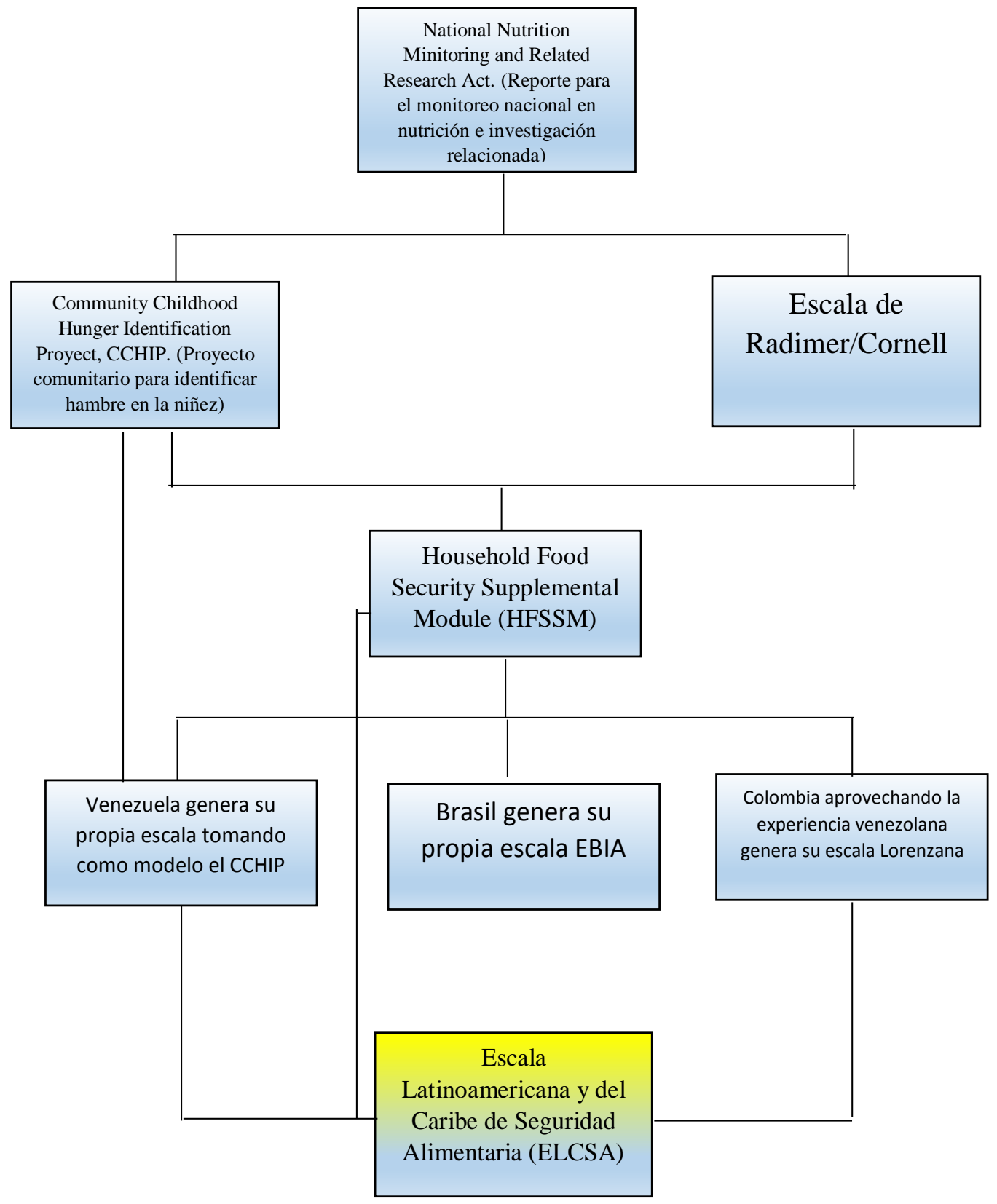

Fuente: elaboración propia 


\section{La ELCSA y su desarrollo}

A partir de su nacimiento, la ELCSA ha estado posicionándose como la opción más adecuada para medir la inseguridad alimentaria en los países de América Latina y el Caribe, sobre todo porque la agilidad en su manejo y pronta obtención de resultados permite su aplicación con objeto de diagnosticar la situación de una localidad, región o país en donde se van a implementar programas de ataque contra la pobreza y, posteriormente, mediante la propia ELCSA, medir objetivamente la operatividad de dichos programas.

Tal vez el reto más importante es que los expertos lograron explotar las cualidades intrínsecas de la Escala y obtuvieron consensos que armonizan la metodología de la ELCSA como una medida válida del nivel de inseguridad alimentaria en los hogares.

La primera versión consensuada de la ELCSA es el resultado de la Primera Conferencia Latinoamericana y del Caribe sobre la Medición de la Inseguridad Alimentaria (CLACMESA I) que se realizó en Antioquía, Colombia en 2007 (Pérez, Álvarez, Segall, Melgar, Nord, 2007, pp. 76 - 164) con la participación de once países. A partir de esta primera conferencia, el desarrollo del uso y afianzamiento de la ELCSA ha sido espectacular. Esta primera versión consensuada fue aplicada por primera vez en Haití en 2007 y considerada de suma importancia porque representaba una prueba de fuego debido a las condiciones de extrema pobreza (Pérez, 2009).

Poco después, la ELCSA comenzó a ser aplicada en estudios locales, como encuestas estatales y nacionales. A nivel nacional, la ELCSA fue aplicada en Colombia en la Encuesta Nacional de Condiciones de Vida (ENCOVI) en 2008.

Los resultados, obtenidos por la ELCSA después de CLACMESA I, confirmaron la validez de la ELCSA, por lo que se realizó la Segunda Conferencia Latinoamericana y del Caribe sobre la Medición de la Inseguridad Alimentaria (CLACMESA II), con el fin de presentar y discutir los resultados obtenidos. Esta conferencia se llevó a cabo en la Universidad de Campinas (UNICAMP), Brasil en el año 2009.

La contribución más importante de esta conferencia fue la decisión de comenzar el proceso de armonización de la ELCSA en encuestas nacionales de la región, para lo cual se propuso la organización del primer taller de armonización, cuyo objetivo es contar con una herramienta que permita hacer mediciones comparables entre los países con mayor afinidad, aunque aplicaciones previas con versiones algo diferentes de la ELCSA ya habían 
mostrado capacidad de comparabilidad entre los países donde se aplicó dicho instrumento (Melgar, 2010).

El taller regional de armonización de la ELCSA se llevó a cabo en 2010 en el Instituto de Salud Pública en Cuernavaca, México; el taller contó con el patrocinio de la FAO (FAO, INSP, 2010) y la representación de México, Guatemala, Nicaragua, Honduras, el Salvador y Perú. El resultado más sobresaliente de este taller fue la versión armonizada de la ELCSA.

Como consecuencia de este taller, fue incorporada a la Encuesta Nacional de Condiciones de Vida 2011 en Nicaragua.

Un segundo taller regional se llevó a cabo en julio de 2011 en Bogotá, Colombia, al cual acudieron representantes de Bolivia, Ecuador, Paraguay, Perú y Colombia (FAO, 2011). En este taller se discutió la versión armonizada de la ELCSA del taller de Cuernavaca, y además se unificaron criterios para someter a la ELCSA a procesos de validación interna y externa.

Otros talleres de alcance nacional fueron organizados en Bolivia y Paraguay. Actualmente, la ELCSA ha sido incorporada a la evaluación del Programa Desnutrición Cero que implementó el gobierno de Bolivia, mientras que en Ecuador fue incorporada esta escala como parte del programa INTI (Intervención Nutricional Territorial Integral).

\section{La ELCSA en México}

El uso de escalas para medir la inseguridad alimentaria en México se remonta a principios de este siglo; se usó una adaptación de la HFSSM en la Sierra de Manantlán, Jalisco, versión que fue validada con éxito. Este estudio formó parte del proyecto PLAN (Planificación Local de la Agricultura y la Naturaleza).

En mayo de 2004, Parás y Pérez reportaron el uso de una versión del HFSSM (basada en la que se utilizó en Brasil), y que fue aplicada a una muestra de la Ciudad de México como parte de una encuesta de opinión pública denominada "El Termómetro Capitalino" (Parás, Pérez, 2004). Un reporte que apareció en la Revista Universidad del Valle de Atemaxac en 2007 menciona la aplicación de una versión de esta escala a adultos mayores en asociación con la Diabetes Mellitus tipo 2, hipertensión y osteoartritis (Dávila, Torres, Márquez, 2007). 
Se reportó también un estudio llevado a cabo en el 2002, publicado en el 2006, donde se usó una versión de la escala Radimir/Cornell aplicada a ancianos pobres de la Ciudad de México (Rivera, Guerrero, 2006).

Existen otros estudios llevados a cabo en la Ciudad de México y Querétaro, en los que se aplicaron versiones adaptadas, principalmente de HFSSM. Este resumen muestra que México, antes de la ELCSA, ya conocía la eficacia y la utilidad de los estudios cualitativos para cuantificar la Inseguridad Alimentaria mediante las escalas.

Las experiencias mencionadas despertaron el interés de los estudiosos e investigadores del sector público y privado por promover y asistir a la Primera Conferencia Latinoamericana y del Caribe sobre la Medición de la Inseguridad Alimentaria (CLACMESA I), celebrada en Antioquía, Colombia, donde los mexicanos fueron protagonistas.

La versión consensuada de la ELCSA, fruto de esta primera conferencia, fue utilizada de inmediato en el estado de Guanajuato en una muestra de 1199 entrevistados, que en 2008 fue aplicada en una encuesta de opinión "Barómetro de las Américas" del Proyecto de Opinión Pública Latinoamericana (LAPOP por sus siglas en inglés) con una muestra de 1511 hogares (Parás, Pérez, 2008). Dicha encuesta se aplicó a nivel nacional y sus resultados fueron publicados y diseminados ampliamente a tal grado que contribuyeron al debate sobre la problemática de la inseguridad alimentaria e influyeron para que el Consejo Nacional de Evaluación de la Política de Desarrollo Social (CONEVAL) adoptara la ELCSA de manera oficial como instrumento para medir uno de los indicadores más importantes de la medición multidimensional de la pobreza adoptada por la SEDESOL en 2008. En ese año, la Encuesta Nacional de Ingreso y Gasto de los Hogares (ENIGH, 2008) incorporó una versión simplificada de la ELCSA en su cuestionario, conocida como Escala Mexicana de Seguridad Alimentaria (EMSA). Sin embargo, para los años 2010, 2012 y 2014, la ENIGH abandonó esta escala y adoptó la ELCSA en su versión emanada de CLACMESA I, posteriormente armonizada en el taller llevado a cabo en Cuernavaca. Otro estudio que adoptó la ELCSA para integrarla a sus encuestas en México es la Encuesta Nacional de Salud y Nutrición (ESANUT), que también se levanta cada dos años.

La mecánica mediante la cual CONEVAL mide el indicador de carencia por acceso a la alimentación, uno de los factores integradores de la pobreza multidimensional, es mediante los resultados de la ELCSA que describen la situación de Seguridad Alimentaria 
identificando tres niveles de Inseguridad Alimentaria en los Hogares (IAH). Para construir estos tres niveles de IAH, la ELCSA tiene 15 ítems (En México, CONEVAL decidió aumentar una pregunta más a la ELCSA (pregunta 9) por lo que ahora consta de 16 preguntas (ver anexo), con opción de respuestas dicotómicas (Sí - No). Son 8 (en México 9) preguntas dirigidas a los adultos del hogar y 7 que involucran a menores de 18 años, por lo tanto, en hogares donde hay menores de 18 años los entrevistados responden a 16 preguntas. De acuerdo a las preguntas contestadas afirmativamente, los puntos de corte para identificar los niveles de IAH se muestran en el cuadro 1.

Cuadro 1. Puntos de corte para la clasificación de la (in)seguridad alimentaria

\begin{tabular}{|c|c|c|c|c|}
\hline \multirow{2}{*}{ Tipo de Hogar } & \multicolumn{3}{|c|}{ Clasificación de la (in)seguridad alimentaria } \\
\cline { 2 - 5 } & Seguridad & $\begin{array}{c}\text { Inseguridad } \\
\text { leve }\end{array}$ & $\begin{array}{c}\text { Inseguridad } \\
\text { moderada }\end{array}$ & $\begin{array}{c}\text { Inseguridad } \\
\text { severa }\end{array}$ \\
\hline $\begin{array}{c}\text { Hogares integrados solamente por } \\
\text { personas adultas }\end{array}$ & 0 & 1 a 3 & 4 a 6 & 7 a 8 \\
\hline $\begin{array}{c}\text { Hogares integrados por personas } \\
\text { adultas y menores de 18 años }\end{array}$ & 0 & 1 a 5 & 6 a 10 & 11 a 15 \\
\hline
\end{tabular}

Fuente: FAO, 2012, p. 68

Para calcular la carencia por acceso a la alimentación, el CONEVAL toma los resultados de la Inseguridad Alimentaria Moderada y los de la Inseguridad Alimentaria Severa; estos dos grados de Inseguridad Alimentaria en los hogares conforman la carencia por acceso a la alimentación. 


\section{Conclusión}

La ELCSA es una herramienta de bajo costo, rápida aplicación y fácil interpretación; además ha demostrado tener una alta validez y confiabilidad en los diversos campos de aplicación, desde el nivel local hasta el nivel nacional, en encuestas gubernamentales, estudios académicos y sondeos de opinión pública. Esta escala tiene gran potencial para seguir contribuyendo a la mejor comprensión de la distribución, causas y consecuencias de la Inseguridad Alimentaria.

En México, la versión de la ELCSA fruto de CLACMESA I ya armonizada se ha aplicado para conocer principalmente la asociación existente de la Inseguridad Alimentaria con variables socio-económicas de grupos de población bien identificados.

En México, a pesar de que dicha herramienta se aplica en dos encuestas periódicas (ENIGH y ENSANUT), y de que el interés por conocerla va en aumento, todavía se encuentra en etapa germinal. El propósito del presente estudio es aumentar el interés en ella. 


\section{Bibliografía}

Álvarez, M; Estrada, A; Melgar, H. (2006). "Validación de la escala de seguridad alimentaria doméstica en Antioquía, Colombia”. En: Salud Pública de México.

CONEVAL (2012). Informe de pobreza en México, el país, los estados y sus municipios 2010. México, D. F. $1^{\text {a }}$ edición, agosto 2012.

CONEVAL (2014). Metodología para la medición multidimensional de la pobreza. $2^{\mathrm{a}}$. Edición. México. D. F.

Dávila, O; Torres, B; Márquez, F. (2007). "Inseguridad Alimentaria y frecuencia de Diabetes Mellitus Tipo 2, hipertensión y osteoartritis en adultos mayores ambulatorios de la estancia diurna del DIF Zapopan, Jalisco". En: Revista Universidad del Valle de Atemaxac. 12 (59).

ENIGH (2000; 2008; 2010; 2012; 2014). Encuesta Nacional de Ingreso y Gasto de los Hogares. México, D. F.

FAO (1996). Declaración de Roma Sobre la Seguridad Alimentaria Mundial. Roma, Italia

FAO, Ohio State University (2012). Informe sobre la aplicación pasada y presente de la Escala Latinoamericana y Caribeña de Seguridad Alimentaria (ELCSA) y otras herramientas similares en América del Norte, Centroamérica y el Caribe. Department of Human Nutrition OSU Extension. Columbus, Ohio.

FAO (2002). Medición y evaluación de la carencia de alimentos y la desnutrición. Simposio Científico Internacional. 26 - 28, junio de 2002. Roma, Italia.

FAO (2006) "Informe de políticas". Número 2. Dirección de Economía Agrícola y del Desarrollo (FAO, junio 2006).

FAO (2008). Metodología de la FAO para medir la privación de alimentos. Dirección de

Estadística de la FAO. Roma, Italia, octubre 2008.

FAO (2011). "Taller de análisis estadístico de ELCSA". Bogotá, Colombia, julio 2011.

FAO (2012). Escala Latinoamericana y Caribeña de Seguridad Alimentaria (ELCSA): Manual de uso y aplicación. Oficina Regional para América Latina y el Caribe. Vitacura, Santiago de Chile.

FAO (2014). El estado mundial de la agricultura y la alimentación 2014. La innovación en la agricultura familiar. Roma, Italia. 
FAO INSP (2010). "Taller de armonización de la ELCSA". Cuernavaca, México, septiembre 2010.

FAO; FIDA; PMA (2015). El estado de la Inseguridad Alimentaria en el mundo 2015. Cumplimiento de los objetivos internacionales 2015 en relación con el hambre: balance de los desiguales progresos. FAO. Roma, Italia.

Fiszbein, A; Giovagnoli, I. (2003). Hambre en Argentina. Documento de trabajo No. 4/03. Disponible en: http://cdi.mecon.gov.ar/biblio/doc/bm/dt/4.pdf Consultado el $30 \mathrm{de}$ junio de 2016.

Hackett, M; Melgar, H; Álvarez, C. (2008). "Internal validity of a Household Food Security Scale is consistent among diverse populations participating in a food supplement program in Colombia". En: BMC Public Health.

Hamilton, W; Cook; J; Thompson, W; Buron, L; Frogillo, E; Olson, C; Eehler, C. (1997). Household Food Security in the United States in 1995. Summary Report of the Food Security Measurement Project. USDA.

Health Canadá (2004). Canadian Community Health Survey. Disponible en: http://www.hc-sc.ca/fn-an/alt_formats/hpfb-dgpsa/pdf/surveill/income_food_secsec_alim-eng.pdf Consultado el 18 de junio de 2016.

Latham, Michael (2002). "Nutrición humana en el mundo en desarrollo". En: Colección FAO: Alimentación y Nutrición No. 29. Organización de las Naciones Unidas para la Agricultura y la Alimentación. Roma, Italia.

Lorenzana, P; Mercado, C. "Measuring household food security in poor Venezuelan households". Public Health Nutr. 2002; 5(6A).

Melgar, H; Álvarez, U; Fonseca, C; Bermúdez, O; Palma, P; Fulladolsa, A; Parás, R. (2010). "Características Psicométricas de la Escala de Seguridad Alimentaria ELCSA aplicada en Colombia, Guatemala y México". En: Revista de Seguranca Alimentar e Nutricional, Campinas. No. 17 (1).

Melgar, H; Zubieta, C; Valdez, E; Whitelaw, B; Kaiser, Lucía (2005). "Validación de un instrumento para vigilar la inseguridad alimentaria en la Sierra de Manatlán, Jalisco". En: Salud Pública de México, vol. 47, núm. 6, México, D. F. 
Melgar, H; Zubieta, A; MkNelly, b; Nteziyaremye, A; Gerardo, M; Dunford, C. (2006). "Household food insecurity and daily per capita food expenditura in Bolivia, Burkina Faso and the Phulippines". Journal of Nutrition.

ONU (1948). Declaración Universal de los Derechos Humanos. 10 de diciembre de 1948.

Parás, P; Pérez, R. (2004). "El rostro de la pobreza: la Inseguridad Alimentaria en el Distrito Federal”. En: Revista Este País, 2004; 158(5). México, D. F.

Parás, Pablo; Pérez, Rafael (2008). "Inseguridad Alimentaria en México". Enfoque, 29 de junio de 2008. Diario Reforma, núm. 743. México, D. F.

Pérez, R; Álvarez, M; Segall, A; Melgar, H; Nord, M. (2007). Memorias. $1^{a}$. Conferencia en América Latina y el Caribe sobre la Medición de la Inseguridad Alimentaria en el Hogar. Antioquía, Colombia.

Pérez, R; Dessaliness, M; Finnigan, M; Pachón, H; Hromi, A; Gupta, N. (2009). "Household food insecurity is associated with childhood malaria in rural Haiti". Journal Nutrition.

Radimer, K; Olson, M; Greene, J; Campbell, C; Habicht, J. (1992). "Understanding hunger and developing indicators to acces it in women and children". En: Journal of Nutrition Education, vol. 24. Sup. 1, january - february 1992.

Rivera, J; Guerrero, A. (2006). "Inseguridad alimentaria en ancianos pobres de la Ciudad de México: Importancia para la Salud Pública y para la agenda Social". En: Nutrición Clínica 9(2).

Shamah - Levy; Mundo, Verónica; Rivera, D. (2014). "La magnitud de la Inseguridad Alimentaria en México: su relación con el estado de la nutrición y con factores socioeconómicos”. En: Salud Pública de México, vol. 56. Supl. 1. Cuernavaca, Morelos, México.

Simoes, Augusto (2001). "Día mundial de la alimentación 2001: Combatir el hambre para reducir la pobreza". En: Comercio exterior, vol. 51, núm. 10. BANCOMEXT. México. 


\section{Anexo 1. ELCSA}

1. En los últimos tres meses, por falta de dinero o recursos ¿alguna vez usted se preocupó de que la comida se acabara?

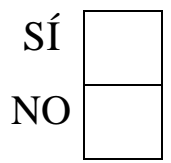

2. En los últimos tres meses, por falta de dinero o recursos ¿alguna vez usted o su familia se quedaron sin comida?

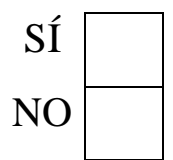

3. En los últimos tres meses, ¿alguna vez se quedaron sin dinero o recursos para obtener una alimentación sana y variada?

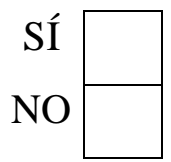

4. En los últimos tres meses por falta de dinero o recursos, ¿alguna vez usted o algún adulto en su hogar tuvo una alimentación basada en muy poca variedad de alimentos?

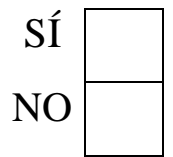

5. En los últimos tres meses por falta de dinero o recursos, ¿alguna vez usted o algún adulto en su hogar dejó de desayunar, comer o cenar?

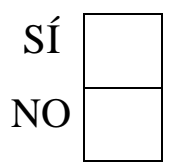

6. En los últimos tres meses por falta de dinero o recursos, ¿alguna vez usted o algún adulto en su hogar comió menos de lo que usted piensa debía comer?

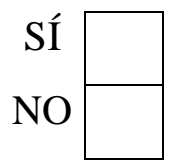

7. En los últimos tres meses por falta de dinero o recursos, ¿alguna vez usted o algún adulto 
en su hogar sintió hambre pero no comió?

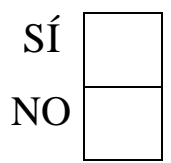

8. En los últimos tres meses por falta de dinero o recursos, ¿alguna vez usted o algún adulto en su hogar sólo comió una vez al día o dejó de comer todo un día?

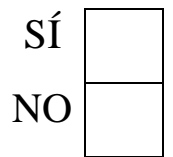

9. En los últimos tres meses por falta de dinero o recursos, ¿alguna vez tuvieron que hacer algo que hubieran preferido no hacer para conseguir comida, tal como mendigar (pedir limosna) o mandar a los niños a trabajar?

\section{Si en el hogar hay menores de 18 años}

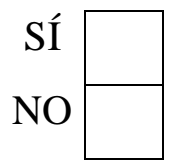

10. En los últimos tres meses por falta de dinero o recursos, ¿alguna vez usted o algún menor de 18 años en su hogar dejó de tener una alimentación sana y variada?

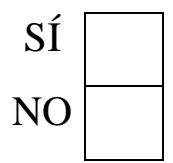

11. En los últimos tres meses por falta de dinero o recursos, ¿alguna vez usted o algún menor de 18 años en su hogar tuvo una alimentación basada en muy poca variedad de alimentos?

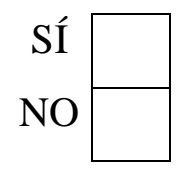

12. En los últimos tres meses por falta de dinero o recursos, ¿alguna vez usted o algún menor de 18 años en su hogar comió menos de lo que debía?

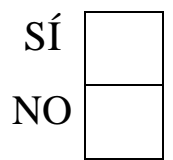

13. En los últimos tres meses por falta de dinero o recursos, ¿alguna vez tuvieron que 
disminuir la cantidad servida en las comidas a algún menor de 18 años en el hogar?

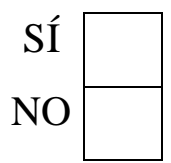

14. En los últimos tres meses por falta de dinero o recursos, ¿alguna vez usted o algún menor de 18 años en su hogar sintió hambre pero no comió?

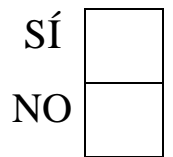

15. En los últimos tres meses por falta de dinero o recursos, ¿alguna vez usted o algún menor de 18 años en su hogar se acostó con hambre?

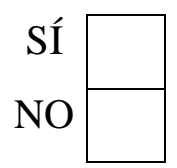

16. En los últimos tres meses por falta de dinero o recursos, ¿alguna vez usted o algún menor de 18 años en su hogar comió una vez al día o dejó de comer todo un día?

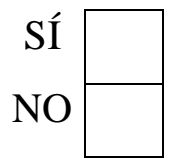

Fuente: ENIGH, 2014. 